\title{
Erklärung der Citate.
}

Eine eingeklammerte (arabische) Zahl vor der (römischen) Bandzabl bezeichnet die Reihe (Serie), zu der der Band gehört. Einige periodische Schriften, in welehen nur zuweilen eine vereinzelte mathematische Arbeit erschienen ist, eind in dieses Verzeichnis nicht aufgenommen worden; das bezügliche Citat im Texte ist dann in hinreichender Ausfübrlichkeit gegeben.

Acta Math.: Acta Mathematica. Zeitschrift herauggegeben von G. MittagLeffler. Stockholm. $4^{0}$. IX, X, XI.

Almeida J.: Journal de physique théorique et appliquée. Fondé par J. Ch. d'Almeida et publié par MM. E. Bouty, A. Cornu, E. Mascart, A. Potier. (2) VI. Paris. Au Bureau du Journal de Physique.

American J.: Americau Journal of Mathematics. Editor S. Newcomb, Associate Editor Th. Craig. Published under the auspices of the Johns Hopkins University. Baltimore. IX, X.

Amst. Versl. en Meded.: Verslagen en Mededeelingen der Koninklijke Akademie van Wetenschappen. Afdeeling Natuurkunde. Amsterdam. (3) III.

Ann. d. Chim. et Phys.: Annales de Chimie et de Physique par MM. Chevreul, Boussingault etc. Paris. Gauthier-Villars. $8^{0}(6)$. X, XI.

Ann. de lEE. Norm.: Annales scientifiques de l'École Normale Supérieure, publiées etc. par un comité de rédaction composé de MM. les maîtres de conférences de l'École. Paris. Gauthier-Villars. $4^{\circ}$. (3) IV.

Annali di Mat.: Annali di matematica pura ed applicata diretti dal prof. Francesco Brioschi colla cooperazione dei professori: L. Cremona, E. Beltrami, E. Betti, F. Casorati. Milano. $4^{\circ}$. (2) XV.

Annals of Math.: Annals of Mathematic8. Ormond Stone, editor. William M. Thornton, associate editor. Office of publication: University of Virginia. B. Westermann and Co. New-York. III.

Arch. f. Art.: Archiv für die Artillerie- und Ingenieur-Officiere des Deutschen Reichsheeres. Redaction: Schröder, Meinardus. 51. Jahrgang. Bd. XCIV. Berlin. Mittler u. Sohn.

Astr. Nachr.: Astronomische Nachrichten, begründet ron H. C. Schumacher. Unter Mitwirkung des Vorstandes der Astronomischen Gesellschaft herausg. von A. Krüger. Kiel. $4^{\circ}$. CXV, 
Batt. G.: Giornale di matematiche ad uso degli studenti delle università italiane pubblicato per cura del Prof. G. Battaglini. Napoli. gr. $8^{\circ}$. XXV.

Belg. Bull.: Bulletins de l'Académie Royale des sciences, des lettres et des beaux-arts de Belgique. Bruxelles. $8^{\circ}$. (3) XIII, XIV.

Belg. Mém: Mémoires de l'Académie Roysle des sciences, des lettres et des beaux-arts de Belgique. Bruxelles, F. Hayez.

Bely. Mém. C.: Mémoires couronnés et antres Mémoires publiés par l'Académie Royale des sciences, des lettres et des beaux-arts de Belgique. Collection in $8^{0}$. Bruxelles. F. Hayez. XL.

Belg. Mein. S. E.: Mémoires couronnés et Mémoires des savants étrangers publiés par l'Académie Royale des sciences, des lettres et des beauxarts de Belgique. Bruxelles. F. Hayez. $4^{\circ}$.

Berl. Abh.: Mathematisch-physikalische Abhandlungen der Kgl. Preussischen Akademie der Wissenschaften zu Berlin. Berlin. $4^{0}$.

Berl. Ber.: Sitzungsberichte der Kgl. Preussischen Akademie der Wissenschaften zu Berlin. Berlin. $8^{0} .1887$.

Berl. phys. Ges. Verh.: Verhandlungen der physikalischen Gesellschaft in Berlis. Berlin. G. Reimer. $8^{\circ}$. 1887. VI.

Bern Mitt.: Mitteilungen der Naturforschenden Gesellschaft in Bern aus dem Jahre 1887. Bern. Huber u. Co.

Besso Per. mat.: Periodico di matematica per l'insegnamento secondario - diretto da D. Besso. Roma. $8^{\circ}$. Il.

Bibl. Math.: Bibliotheca Mathematica, herausgegeben von G. Eneström: Stockholm 1887. (2) I.

Böklen Mitt: Mathematisch-naturwissenschaftliche Mitteilungen herausgegeben von Dr. O. Böklen. Tübingen. Fr. Fues. I, II.

Bologna Mem.: Memorie dell' Accademia Reale di scienze dell' Istituto di Bologna. Bologna. 4". (4) VII, VIII.

Bologna Rend.: Rendiconti dell' Accademia Reale di scienze dell' Istituto di Bologna. Bologna.

Bonc. Bull.: Bulletino di bibliografia e di storia delle scienze matematiche e fisiche pubblicato da B. Boncompagni. Roma. $4^{\circ}$. XIX, XX.

Bord. Mém.: Mémoires de la Société des sciences physiques et naturelles de Bordeaux. Bordeaux. Paris. $8^{\circ}$.

Brit. Ass. Rep.: Reports of the meeting of the British Association for the - advancement of science. London. gr. 8?.

Brux. Ann.: Annales de l'Observatoire Royal de Bruxelles, publiées aux frais de l'Etat. Bruxelles. F. Hayez. $4^{\circ}$.

Brux. S. sc.: Annales de la société scientifique de Bruxelles. Bruxelles. F. Hayez. (Doppelt paginirt, unterschieden durch $\mathbf{A}$ und B.). XI.

Cambr. Proc.: Proceedings of the Cambridge Philosophical Society. Cambridge. VI.

Cambr. Trans.: Transactions of the Philosophical Society of Cambridge. Cambridge.

Casop.: Casopis; Zeitschrift zur Pflege der Mathematik und Physik, redigirt mit besonderer Rücksicht auf Studirende der Mittel- und Hochschulen von F. J. Studniěka, herausgegeben vouı Vereine böhmischer Mathematiker in Prag. Prag. $8^{0}$. (Böhmisch.) XVI.

Centralb. d. Bauverw.: Centralblatt der Banverwaltung. Herausgegeben im Ministerium der öffentlichen Arbeiten. Redacteure O. Sarrazin und K. Scbäfer. Berlin. Ernst u. Korn. VII. 
Chark. Ges.: Sammlung der Mitteilungen und Protokolle der mathematischen Gesellschaft in Charkow. (Russisch.)

Civiling.: Der Civilingenieur. Organ des sächsiscben Ingenieur- und Architekten-Vereins. Unter Mitwirkung etc. herausgegeben von Dr. E. Hartig. Jabrg. 1887. (2) XXXIII. Leipzig. Arthur Felix. $4^{0}$.

C. $R$.: Comptes Rendus hebdomadaires des séances de l'Académie des Seiences. Paris. $4^{\circ}$. CIV, CV.

Darb. Bull.: Bulletin des sciences mathématiques, rédigé par MM. G. Darboux, J. Hoüel 'et $J$. Tannery avec la collaboration de MM. André, Battaglini etc. Paris. Gauthier-Villars. $8^{\circ}$. (2) XI.

Delft Ann. d. l'Éc. Polyt.: Annales de l'École Polytechnique de Delft. Leiden. E. J. Brill. III.

Deutsche Bauztg.: Deutsche Bauzeitung. Verkündigungsblatt des Verbandes deutscher Architekten- und Ingenieurvereine. Redacteure K. E. O. Fritsch und E. W. Büsing. Berlin. E. Toeche. XXI.

Dorpat. Naturforscher Ges. Ber.: Sitzungsberichte der Dorpater Naturforscher-Gesellscbaft. Dorpat.

Dublin Trans.: Transactions of the Royal Irish Academy. Dublin. XXIX.

Edinb. M. S. Proc.: Proceedings of the Edinburgh Mathematical Society. V.

Edinb. Proc.: Proceedings of the Royal Society of Edinbargh. Edinburgh. $8^{\circ}$. XIII, XIV.

Edinb. Trans.: Transactions of the Royal Society of Edinburgh. Edinburgh. $4^{0}$. XXXIII.

Ed. Times: Mathematical questions, with their solutions from the "Educational Times" with many papers and solutions not published in the „Educational Times.“ Edited by W. J. C. Miller. London. $8^{\circ}$. Francis Hodgson. XLVI, XLVII.

Erlang. Ber.: Sitzungaberichte der physikalisch-medicinischen Societät zu Erlangen. Erlangen. $8^{0}$.

Exner Rep.: Repertorium der Physik herausgegeben von Exner. München und Leipzig. gr. $8^{\circ}$. XXIII, XXIV.

Flammarion, Rev. d'Astr.: I'Astronomie. Revue d'astronomie populaire, do météorologie et de physique du globe, exposant les progrès de la science pendant l'année. Paris.: Gauthier-Villars. gr. $8^{\circ}$. VI.

Franc. Ass.: Association Française pour l'avancement des sciences naturelles. ('T'oulouse.)

Génie civ.: Le Génie civil. Revue, générale hebdomadaire des industries françaises et étrangères. Paris. X, Xl.

Gen. Mém.: Mémoires de la société de physique et d'histoire naturelle de Genève. Genève. $4^{0}$. Librairie H. Georg. XXIX.

Genova G.: Giornale della Società di letture e conversazioni scientifiche in Genova. $8^{\circ} .1887$.

Gott. Abh.: Abhandlungen der Kgl. Gesellschaft der Wissenschaften zu Göttingen. Göttingen. $4^{0}$. XXXIV.

Gött. N.: Nachrichten von der Königlichen Gesellschaft der Wissenschaften und der Georg-August-Universität zu Göttingen. Göttingen. $8^{\circ}$. 1887 .

Hamb. Mitt.: Mitteilungen der Hamburger Mathematischen Gesellischaft. Hamburg. $8^{0}$.

Hannov. Zeitschr.: Zeitschrift des Architekten- und Ingenieurvereins zu Hannover, redigirt von Keck. Hannover. Schmorl u. Seefeld. XXXIII. 
Helsingf. Vet. soc. Acta: Acta societatis scientiarum Fennicae. $4^{\circ}$.

Helsingf. Vetensk. soc. Öjv.: Öfversigt af finska vetenskaps-societetens förhandlingar. Helsingfors. $\rangle^{0}$.

Hoffmann 2.: Zeitschrift für mathematischen und naturwissenschaftlichen Unterricht. Unter Mitwirkung von Fachlehrern herausgegeben von J. C. V. Hoffmann. Leipzig. Teubner. $8^{\circ}$. XVIII.

Hoppe Arch.: Archiv der Mathematik und Pbysik mit besonderer Berücksichtigung der Bedürfnisse der Lehrer an den höheren Lehranstalten, gegründet von J. A. Grunert, fortgesetzt von R. Hoppe. Leipzig C. A. Koch. $8^{\circ}$. (2) IV, V, VI.

J. de l'Éc. Pol.: Journal de l'École Polytechnique, publié par le conseil d'instruction de cet établissement. Paris. Gauthier-Villars. $4^{0}$. Cah. LVI, LVII.

J. de Math. spec.: Journal de Mathématiques spéciales etc. publié sous la direction de Longchamps, Lucien Lévy. Paris. Delagrave. (3) I.

J. für Math.: Journal für die reine und angewandte Mathematik. In zwang. losen Heften. Herausgegeben von L. Kronecker und K. Weierstrass. Berlin. G. Reimer. $4^{\circ}$. IC, C, Cl, CII.

J. Hopkins circ.: Johns Hopkins University Circulars. Baltimore.

Ing. civ.: Mémoires et Compte Rendu des travaux de la Société des Ingénieurs civils. Paris. II.

Jordan Z.f. V.: Zeitschrift für Vermessungswesen. Organ des deutschen Geometervereins. Unter Mitwirkung von C. Steppes und R. Gerke herausgegeben von W. Jordan. Stuttgart. XVI.

Journ. de Math.: Journal de Mathématiques pures et appliquées, fondé en 1836 et publié jnsqu'en 1874 par J. Liouville. Publié pair C. Jordan avec la collaboration de G. Halphen, E. Laguerre, M. Lévy, A. Mannheim, E. Picard, H. Resal. Paris. (4) III.

Kazan Ber.: Sitzuogsberichte der mathematiscben Section des Naturforschenden Vereins zu Kazan.

Kazan Ges.: Sammlung der Mitteilungen der physikalisch-mathematischen Gesellschaft zu Kazan. (Russisch.) V, VI.

Kazan Nachr.: Nachr. der Kaiserlichen Universität zu Kazav.

Kiew Nachr.: Nachrichten der kaiserlichen Universität zu Kiew. (Russisch.) Kjob. Skrift.: Schriften der Kopenhagener Akademie. Kopenhagen. (6) 11 .

Kopenh. Overs.: Oversigt over det Kongelige Danske Videnskabernes Selskabs Forhandlinger. Kopenhagen.

Krak. Ber.: Sitzungsberichte der mathematisch-naturwissenschaftlichen Section der Krakauer Akademie. Krakau. (Polnisch.) XV.

Krak. Denkschr.: Denkschriften der Krakauer Akademie der Wissenschaften. Krakau. (Polnisch.) XIII.

Leipz. Abh.: Abhandlungen der Königl. Sächsischen Gesellschaft der Wissenschaften zu Leipzig. Leipzig. XIII.

Leipz. Ber.: Berichte über die Verhandlungen der Königl. Sächsischen Gesellschaft der Wissenschaften zu Leipzig. Leipzig.

Liège Mem.: Mémoires de la Société Royale des sciences de Liège. (2) XII, XIII.

Lisb. J.: Jornal de Sciencias Mathematicas, Physicas e Naturales publicados sob os anspicios da Academia Real das Sciencias de Lisboa. Lisboa.

Lisb. Mem.: Memorias da Academia Real das Sciencias de Lisboa. Lisboa. 
Lomb. Ist. Rend.: Reale Istituto Lombardo di scienze e lettere. Rendiconti. Milano. $8^{\circ}$. (2) $\mathrm{XX}$.

Lond. M. S. Proc.: Proceedings of the London Mathematical Society. London. $8^{\circ}$. XVIII.

Lond. Phil. Trans.: Philosophical Transactions of the Royal Society of London. London. $4^{\circ}$. CLXXVIII.

Lond. R. S. Proc.: Proceedings of the Royal Society of London. London. $8^{0}$ XLII, XLIII.

Lund Arsskr.: Acta universitstis Lundensis. Lunds Universitets Årsskrift. Lund.

Manch. Mem.: Memoirs of the fitterary and philosophical Society of Manchester. Manchester.

Mar. J.: Marine Journal. (Russisch.)

Math. Ann.: Matbematische Annalen. In Verbindung mit C. Neumann begründet durch R. F. A. Clebsch. Untèr Mitwirkung der Herren P. Gordan, C. Neumann, K. VonderMühll gegenwärtig herausgegeben von F. Klein und A. Mayer. Leipzig. Teubner. $8^{\circ}$. XXVIII, XXIX, XXX.

Mathesis: Mathesis, Recueil mathématique à l'usage des écoles spéciales et des établissements d'instruction mayenne publié par P. Mlansion et J. Neuberg. Gand. Hoste. Paris. Gauthier-Villars. 80. VII.

Jfess.: The Messenger of Mathematics, edited by C. Taylor and J. W. L. Glaisher. London and Cambridge. Macmillan and Co. $8^{\circ}$. (2) XVI, XVII.

Met. Zeitschr.: Meteorologische Zeitschrift. Berausgegeben von der Oestreich. Gesellschaft für Meteorologie und der deutschen Meteorol. Gesellschaft, redigirt von J. Hann u. W. Koeppen. Berlin. V.

Mitt. üb. Art. u. Genie: Mitteilungen über Gegenstände des Artillerie- und Genie - Wesens Herausgegeben vom K. K. technischen u. administrativen Militär-Comité. Wien. R. v. Waldheim. $8^{0}$. XVIII.

Modena Mem.: Memorie della Accademia Reale di Modena. Modena. (2) $\mathbf{V}$.

Mosk. Math. Samml.: Mathêmatische Sammlung herausgegeben von der Mathematischen Gesellschaft in Moskau. (Russisch.) XIII.

Mosk. Nachr.: Nachrichten der Mosksuer Universität. Moskau. (Russiscb).

Münch. Abh.: Abhandlungen der Kgl. Bairischen Gesellschaft der Wissenschaften zu München. Zweite Klasse. München. XVI.

Münch. Ber.: Sitzungsberichte der Kgl. Bairischen Akademie der Wissenschaften zu München. München. $8^{\circ}$. X VII.

Nap. Rend.: Rendiconti dell' Accademia delle scienze fisiche e matema. tiche di Napoli. Napoli. $4^{\circ}$. (2) I.

Nature: Nature, a weekly illustrated journal of science. London and New York. Macmillan and Co. XXXVI, XXXVII.

Nérl. Arch.: Archives Néerlandaises des sciences exactes et naturelles, publiées par la Société Hollandaise des sciences à Harlem. La Haye. $8^{\circ}$. XXII.

Nieuw Arch.: Nieuw Archief voor wiskunde nitgegeven door het Wiskundig Genootschap. Amsterdam. $8^{\circ}$. XIV.

Nouv. Ann.: Nouvelles Annales de mathématiques. Journal des candidats aux Ecoles Polytechnique et Normale, rédigé par MM. Gerono et Ch. Brisse. Paris. $8^{\circ}$. (3) VI. 
Nuovo Cimento: II Nuovo Oimento. Giornale fondato per la fisica e la chimica da C. Matteucci e R. Piria, continuato per la fisica esperimentale e matematica da E. Betti e R. Felici. Pisa. Salvioni. (3) XXI, XXII.

Odessa Ges.: Denkschriften der mathematischen Abteilung der nenrussischen Gesellschaft der Naturforscher. (Russisch). VIII.

Odessa Nachr.: Nachrichten von der Universität Odessa. Odessa.

Palermo Rend.: Rendiconti del Circolo Matematico di Palermo. Palermo. I.

Padova Atti: Atti della Reale Accademia di scienze, lettere ed arti di Padova. Padova.

Petersb. Abh.: Abhandlungen der Kais. Akademie der Wissenschaften zu St. Petersburg. Petersburg. LV.

Phys. Ges. St. Pet.: Journal der physiko-chemischen Gesellschaft zu St.Petersburg. XVIII, XIX.

Phys. Math. Wiss.: Die physiko-mathematischen Wissenschaften. Journal der reinen und angewandten Mathematik, Astronomie und Physik, herausgegeben von W. W. Bobynin. Moskau. (Russisch.) II.

Phil. Mag.: The London, Edinburgh and Dublin philosophical Magazine and journal of science, by Kane, 'Thomson, Francis. London. $8^{\circ}$. (5) XXII-XXIV.

Poske Z.: Zeitschrift für den physikalischen und chemischen Unterricht. Unter der besonderen Mitwirkung von E. Mach und B. Schwalbe, herausgegeben von F. Poske. Berlin. J. Springer. I.

$P r .=$ Programmabhandlung, Gymn. = Gymnasium, Realgymn. $=$ Realgym nasium, etc.

Prag. Abh.: Abhandlungen der Königl. Böhmischen Gesellschaft der Wissenschaften. Prag. Selbstverlag der Königl. Böhmischen Gesellschaft. $4^{0}$. VII.

Prag. Ber.: Sitzungsberichte der Kgl. Böhmischen Gesellschaft der Wissenschaften. Prag. 8". 1887.

Quart. J.: The Quarterly Journal of pure and applied Mathematics. Edited by N. M. Ferrers, A. Cayley, J. W. L. Glaisher, A. R. Forsyth. London. $8 "$. XXII.

Rev. d'Art.: Revue d'Artillerie paraissant le $\$ 5$ de chaque mois. Paris. 80. XXIX, XXX.

Rom. Acc. L. Rend.: Atti della Reale Accademia dei Lincei. Reudiconti. Roma $4^{0}$. (4) III.

Rom. Acc. L. Mem.: Memorie della Reale Accademia dei Lincei. Roma. gr. $4^{\circ}$. (4) IV.

Rom Acc. P. d. N. L.: Atti della Accademia Pontifica dei Nuovi Lincei. Roma. $4^{0}$. XXXVII, XXXVIII.

Schlömilch Z.: Zeitschrift für Mathematik und Physik, herausgegeben unter verantwortlicher Redaction von Schlömilch, Kahl und Cantor. Leipzig. Teubner. $8^{\circ}$. XXXII.

Hl. A.: Historisch-literarische Abteilung (besonders paginirt).

Schweiz. Bauztg.: Revue Polytechnique; Schweizerische Bauzeitung, Wochenschrift für Bau-, Verkehrs- und Maschinentechnik, Organ des S'chweizerischen Ingenieur- und Architekten-Vereins etc. Herausgegeben von Waldner. IX, $\mathrm{X}$.

Sill. J.: The American Journal of science. Editors: J. D. and E. S. Dana.

S. M. F. Bull.: Bulletin de la Société Mathématique de France publié par les secrétaires. Paris. $8^{\circ} . \mathbf{X} \nabla$.

Stockh. Handl.: Handlingar af Kongl. Svenska Vetenskaps-Akademiens. Stockholm. 
Stockh. Öfv.: Öfversigt af Kongl. Svenska Tetenekaps-Akademiens Förhandlingar. Stockholm. XLIV.

Stockh. Vetensk. Bihang: Bihang till Kongl. Svenska Vetensksps-Akademiens. handlingar, Stockholm. $8^{0}$.

Techn. Bl.: Technische Blätter, Vierteljahrschrift des deutschen Polytechnischen Vereins in Böhmen, redigirt von Ed. Maiss. Prag. XIX.

Techn. Inst. St. Pet.: Die Mitteilungen des Technologischen Instituts in St.-Petersburg. (Russisch.)

Teixeira J.: Jornal de Sciencias Mathematicas e Astronomicas publicado pelo Dr. F. Gomes Teixeira. Coimbra. 80. VIII.

Torino Atti: Atti della Reale Accademia di Torino. Torino. $8^{\circ}$ XXII, XXIII.

Torino Mem.: Memorie della Reale| Accademia delle scienze di. Torino. Torino.

Toulouse Ann.: Annales de la Faculté des Sciences de Toulouse pour les sciences mathématiques et les sciences physiques, publiées par un comité de rédaction composé des professeurs de mathématiques, de physique et de chimie de la faculté etc. Paris. Gauthier-Villars. I.

Toul. Mém.: Mémoires de l'Académie des sciences, inscriptions et belleslettres de Toulouse. Toulouse. Douladoure-Privat. $8^{0}$. (8) IX.

Ups. N. Act: Nova Acta Regiae Societatis Scientiarum Upsaliensis. Upsala. $4^{\circ}$.

Ven. At.: L'Ateneo Veneto. Rivista mensile di scienze, lettere ed arti diretta da A. S. de Kiriaki e L. Gambari. Venezia. (9) II, (10) I, II.

Ven. Ist. Atti: Atti del Reale Istituto Veneto di scienze, lettere ed arti. Venezia. $8^{0}$. (6) V.

Ven. Ist. Mem.: Memorie del Reale Istituto Veneto di scienze, lettere ed arti. Venezia. XXII.

Wash. Bull.: Bulletin of the Philosophical Society of Washington. X.

Wiedemann Ann.: Annalen der Physik und Chemie. Unter Mitwirkung der Physikalischen Gesellschaft zu Berlin und insbesondere des Herrn H. v. Helmholtz herausgegebeo von G. Wiedemann. Leipzig. Barth. $8^{0}$. (2) XXIX, XXX, XXXI, XXXII.

Wiedemann Beibl.: Beiblätter zu den Annalen der Physik und Chemie. Herausgegeben upter Mitwirkung befreundeter Physiker von G. und E. Wiedemann. Leipzig. Barth. $8^{\circ}$.

Wien. Anz.: Anzeigen der Kaiserlichen Akademie der Wissenschaften zu Wien. Mathematisch-naturwissenschaftliche Klasse. Wien. $8^{0} .1887$.

Wien. Bauztg.: Allgemeịne Bauzeitung gegründet von Chr. L. Förster. Redigirt unter Mitwirkung etc. von A. Köstlin. Wien. R. v. Waldheim. LII.

Wien. Ber.: Sitzungsberichte der mathematisch-naturwissenschaftlichen Klasse der Kaiserl: Akademie der Wissenschaften zu Wien. Zweite Abteilung. Wien. $8^{\circ}$. XCV, XCVI.

Wien. Denkschr.: Denkschriften der Kaiserl. Akademie der Wissenschaften in Wien. Mathematisch-naturwissenschaftliche Klasse. Wien. $4^{0}$. LIII.

Wochenbl. für Bauk: Wochenblatt für Baukunde. Organ der Architektenu. Ingenieurvereine von Bayern, Elsass-Lothringen, .... Herausgegeben von Fr. Scheck. Frankfurt a. Main.

W. Oestr. Ing. u. Arch.: Wochenschrift des Oesterreichischen Ingenieurund Architekten-Vereins. Redacteur P. Kortz. Wien. XII. 
Wolf Z.: Vierteljahrsschrift der naturforschenden Gesellschaft in Zürich von R. Wolf. Zürich. $8^{\circ}$. XXXII.

Z. f. Bauwesen: Zeitschrift für Bauwesen, herausgegeben im Ministerium der öffentlichen Arbeiten. Redacteure O. Sarrazin u. K. Schäfer. Berlin. Ernst u. Korn. XXXVII.

Z. Oestr. Ing. u. Arch.: Zeitechrift des Oesterreichischen Ingenieur- u. Architekten-Vereins. Redacteur J. Melan. Wien. XXXIX.

Z. dtsch. Ing.: Zeitschrift des Vereins deutscher Ingenieure, herausgegeben von Th. Peters. J. Springer. Berlin. $4^{0}$. XXXI.

Zeuthen T.: Tidsskrift for Mathematik. Udgivet af J. P. Gram og B. G. Zeuthen. Kopenhagen. $8^{\circ}$. (5) IV, V. 